\title{
Electron Collisions with Laser Cooled and Trapped Metastable Helium Atoms: Total Scattering Cross Sections
}

\author{
L. J. Uhlmann, R. G. Dall, A. G. Truscott, M. D. Hoogerland, ${ }^{*}$ K. G. H. Baldwin, and S. J. Buckman ${ }^{\dagger}$ \\ Atomic and Molecular Physics Laboratories, Research School of Physical Sciences and Engineering, Australian National University, \\ Canberra, Australian Capital Territory 0200, Australia \\ (Received 12 January 2005; published 3 May 2005)
}

\begin{abstract}
Absolute measurements of total scattering cross sections for low energy $(5-70 \mathrm{eV})$ electrons by metastable helium $\left(2^{3} S\right)$ atoms are presented. The measurements are performed using a magneto-optical trap which is loaded from a laser-cooled, bright beam of slow $\mathrm{He}\left(2^{3} S\right)$ atoms. The data are compared with predictions from convergent close coupling and $R$ matrix with pseudostate calculations, and we find good agreement between experiment and theory.
\end{abstract}

DOI: 10.1103/PhysRevLett.94.173201

PACS numbers: 34.80.Dp

The measurement of absolute scattering cross sections for excited species has long been of interest to both the scattering community and to those modeling the behavior of gas discharges. Excited atoms, particularly those in metastable states, are known to have extremely large scattering cross sections. Thus, although they may be present, for example, in only a small equilibrium population in a discharge environment, the large scattering cross sections, and low excitation and ionization potentials, can dramatically affect the behavior of the discharge. Excited rare-gas metastable atoms are prime examples of this, as they have large internal excitation energies, low binding energies, and high dipole polarizabilities, such that their low energy interactions with electrons are characterized by extremely large cross sections, in excess of $100 \AA^{2}$ [1].

In addition to the potential application of such measurements, there is an interest in the accurate description of such scattering processes by contemporary scattering theory. Helium metastables, in particular, have been the subject of significant interest. In contrast to corresponding ground state comparisons, there are significant differences for metastable helium between recent experiments and state-of-the-art calculations such as the convergent close coupling (CCC) or $R$ matrix with pseudostate (RMPS) techniques. Important examples of these differences occur for the total ionization cross section [2,3] and the total cross section for the $2^{3} S-2^{3} P$ excitation [4]. In these and other cases, theory generally predicts cross sections that are smaller than those measured, and, in the case of ionization, the differences are about a factor of 2. This has drawn into question, for instance, whether the use of the frozen core approximation in the CCC approach is reasonable, and has prompted Bray et al. [5] to suggest that the problem "needs resolution urgently." Previous measurements of the total cross section for $\mathrm{He}\left(2^{3} S\right)$ date back to the 1970s and include both absolute [6] and relative measurements [7], although they are only at low energies $(<10 \mathrm{eV})$ and the uncertainties are greater than $50 \%$.
Measurements of absolute cross sections for such species are notoriously difficult, mainly as a result of the absence of reliable, high-density sources of excited atoms $[8,9]$. Experiments at the exit of discharge sources are most common but are typically plagued with background problems from electrons, ions, and photons. They are also contaminated by ground state species, with typically only one in $10^{5}$ of the atoms in the excited state. Perhaps the most successful and cleanest sources to date have been the charge-transfer devices $[10,11]$, which have been used for measurements of total ionization cross sections for neon metastables [10] and total cross sections for electronic excitation of He metastables [11].

In the present work, we have used laser-cooling and trapping techniques to produce a localized, high-density source of metastable $\mathrm{He}\left(2^{3} S\right)$ atoms for electron collision experiments. The atoms are located far from the discharge source at the center of a 3D magneto-optical trap (MOT) which is loaded from a bright beam of $\mathrm{He}\left(2^{3} S\right)$ atoms, also produced by laser-cooling techniques. Atom trap-based techniques have been used previously for electron collision studies, but not with metastable excited atomic species such as $\mathrm{He}\left(2^{3} S\right)$. Previous experiments have involved total cross section measurements on $\mathrm{Rb}$ [12] and Cs [13] and total ionization measurements on $\mathrm{Rb}$ [14]. A similar experimental approach has been used here, although the light mass and strong Penning ionization of the He atoms provides for substantially greater difficulties in maintaining significant metastable atom densities.

The metastable $\mathrm{He}\left(2^{3} S\right)$ beam line and trap have been described elsewhere [15]. The metastable atoms are produced in a de discharge and are manipulated by three stages of laser cooling (transverse, longitudinal, and compression) to form a slow $\left(<100 \mathrm{~ms}^{-1}\right)$, intense $\left(10^{10}\right.$ atoms s$\left.^{-1}\right)$ beam. Slow atoms from the beam line are loaded into the trap where they are further cooled and trapped by three pairs of orthogonal, counterpropagating laser beams, in conjunction with a quadrupole magnetic 
field. The lasers are tuned to the $2^{3} S_{1}-2^{3} P_{2}$ transition at a wavelength of $1083 \mathrm{~nm}$. At most, about $2 \times 10^{9}$ atoms can be stored in a cloud of $\sim 8 \mathrm{~mm}$ diameter, corresponding to an atomic density of $\sim 5 \times 10^{9} \mathrm{~cm}^{-3}$ with a temperature of about $1 \mathrm{mK}$. This cloud exists in an ultrahigh high vacuum environment that is located $3 \mathrm{~m}$ from the discharge source, and the ratio of $\mathrm{He}\left(2^{3} S\right): \mathrm{He}\left(1^{1} S\right)$ is about 100:1. Using optical molasses techniques, the trapped atoms are further cooled to $\sim 300 \mu \mathrm{K}$, where they have an average ballistic velocity of $\sim 1 \mathrm{~ms}^{-1}$. The relative number of trapped atoms is monitored by detecting the fluorescence, at $1083 \mathrm{~nm}$, with a fast, low-noise photodiode. We note that the excited atom number density achieved with the present setup is at least an order of magnitude larger than other more conventional techniques and is free of the high ground state concentrations and large backgrounds of electrons, ions, and photons that plague conventional discharge sources.

Electrons are introduced into the trap from a purposebuilt, pulsed, low energy electron gun. The electrons are obtained by thermionic emission from a $\mathrm{BaO}$ cathode and accelerated into the trap chamber by electrostatic electron optical elements. The $\mathrm{cw}$ beam currents for this gun vary between 10 and $150 \mu \mathrm{A}$, depending on the incident energy, and the energy spread is about $0.3 \mathrm{eV}$. While the profile of the electron beam varies with energy, it is typically slightly larger than the size of the MOT, and the current density, measured across the profile of the MOT with a scanning wire probe in conjunction with a Faraday cup, is relatively uniform.

After loading the trap and molasses cooling, the laser beam used for longitudinal cooling is blocked with a shutter, the trap laser and magnetic fields are turned off, and the $e$ beam is very quickly pulsed on. A typical timing sequence is shown in Fig. 1. The electron beam is pulsed on within $1 \mathrm{~ms}$ of the magnetic field coils and laser beams

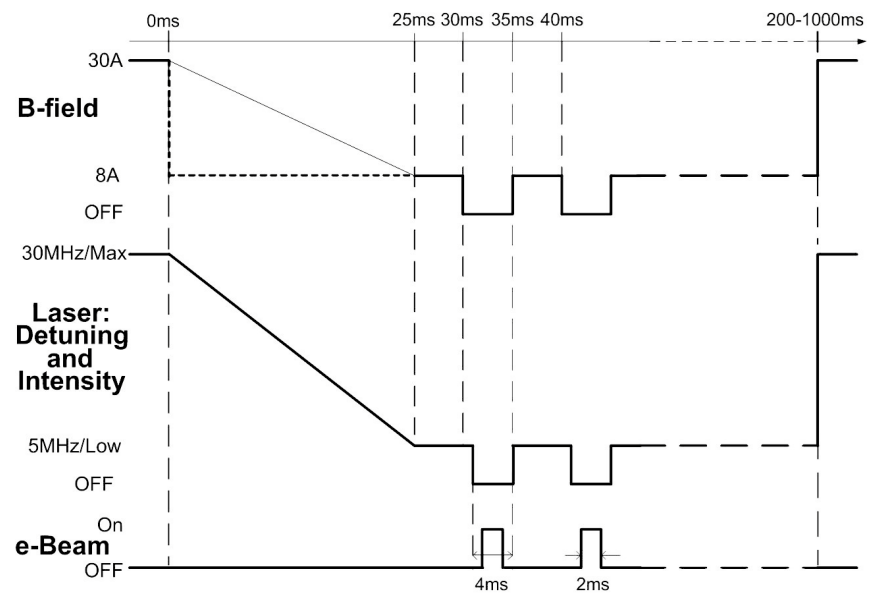

FIG. 1. Timing sequence of the magnetic and optical trapping fields and electron gun pulse. Note that the linewidth of the cooling transition $\left(2^{3} S-2^{3} P\right)$ is $1.6 \mathrm{MHz}$. being turned off, and is typically on for a few ms. A special compensating circuit [16] is employed to drive the $B$ field to zero within $1 \mathrm{~ms}$.

In the present experiments, a "release and recapture" scheme is used to optimize the data collection efficiency. In this scheme, atoms are released from the trap and allowed to ballistically expand for some short time before the trapping fields are turned on again. Those atoms that remain within the influence of the MOT fields are then retrapped. Ideally, ignoring collisional losses, if the atoms were cold enough, and if the trap-off time short enough, all of the atoms would be recaptured when the MOT is turned back on. In practice, this is not the case and the release recapture cycle can typically be repeated between 20 and 100 times before most of the atoms are lost from the trap (see Fig. 1). If, in addition to this cycle, the electron beam is pulsed on during the brief trap-off time within each release/recapture cycle, some fraction of the atoms will be lost due to electron collisions. By monitoring the loss of atoms with and without the electron beam present, the total electron collision cross section can be determined.

By modulating the electron beam with alternate trap loadings, the trap loss rate due to electron collisions $\left(\Gamma_{e}\right)$, together with that due to the natural loss rate from the trap $\left(\Gamma_{0}\right)$, can be measured. The total cross section $(\sigma)$ is derived from the expression

$$
\Gamma_{e}=\frac{\sigma J}{e},
$$

where $J$ is the absolute electron current density and $e$ the electronic charge. $\Gamma_{e}$ is determined from analyzing the rate of change of trapped atoms, $N(t)$, which in the present case is given by

$$
\frac{d N(t)}{d t}=L-\left(\Gamma_{0}+\Gamma_{e} f\right) N(t)-\frac{\beta}{V_{\mathrm{eff}}}(N(t))^{2},
$$

where $L$ is the trap load rate, $f$ is the electron beam duty cycle, $\beta$ is the Penning loss rate [17], and $V_{\text {eff }}$ is the volume of the atomic cloud. In the present case, $L=0$ as the slow beam is shut off during the experimental cycle. $\Gamma_{0}$ is obtained from the trap decay measurement without the $e$ beam present. A typical decay curve measurement is shown in Fig. 2. The requirement to account for Penning ionization processes, which are a significant loss mechanism for metastable rare-gas traps, is an additional complication which must be considered in the present analysis, but not, for example, in alkali traps.

This technique assumes that, in principle, all of the atoms that interact with an electron are ejected from the trap. However, the need to maximize the electron interaction time, by having as little dead time as possible between the end of the electron beam pulse and the turn on of the retrapping fields, competes with the requirement that even those atoms that are involved in small-angle electron scattering events (and as a result have a small amount of 


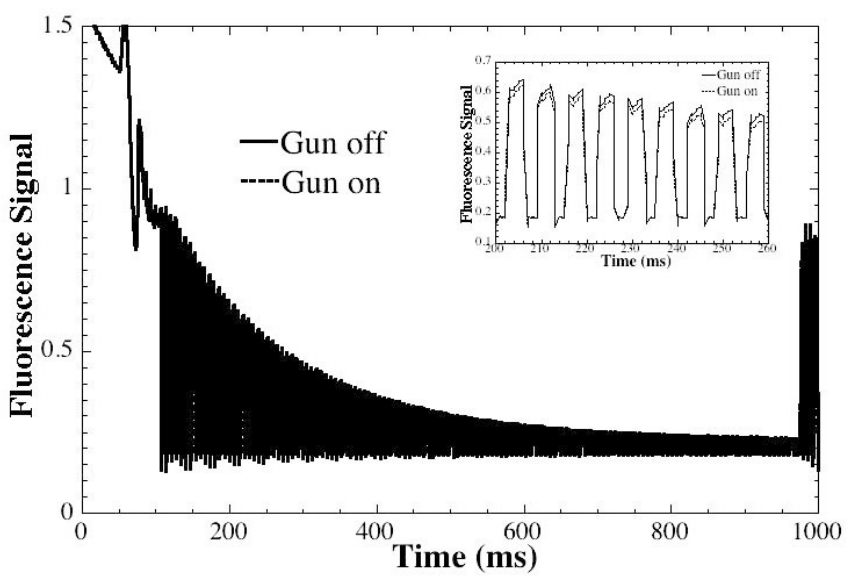

FIG. 2. Fluorescence decay signal for one loading of the trap. The release/recapture sequence can be more clearly seen in the inset, with expanded time scale, as can the difference in the signal between the electron gun on (upper trace) and electron gun off (lower trace) cycles.

momentum transferred to them) still leave the trap. The smallest dead time that the present timing sequence allows is about $200 \mu \mathrm{s}$, and this was a typical value used for the present experiments. The scattered atom velocity can be readily calculated as a function of incident electron energy and scattering angle, and, for a given dead time between the electron pulse and retrapping, an effective minimum scattering angle can be determined simply from the size of the trap laser beam. Below this angle, scattering events will not lead to atom loss from the trap and will therefore not contribute to the total cross section determination. However, the retrapping capacity is greatly reduced during the initial stages (the first few hundred milliseconds) since the intensity and detuning of the trapping lasers, and the magnitude of the magnetic field, is such that the effective trapping velocity is less than $10 \mathrm{~m} / \mathrm{s}$ (typical peak values during trap loading are $70 \mathrm{~m} / \mathrm{s}$ ). Any atoms with larger velocities than this are lost from the trap during this initial phase of retrapping. From the experimental parameters, we estimate that the minimum scattering angle that is probed for the present measurements is less than $3^{\circ}$ at $5 \mathrm{eV}$ and less than $1^{\circ}$ at $75 \mathrm{eV}$. While we expect that the differential cross sections for many of the scattering processes are likely to be significantly enhanced at forward angles, the weighted contribution from those events that are not measured in the present experiment should be less than a few percent, i.e., considerably less than the uncertainty in the measurement. To check this, we have made a number of measurements, at an energy of $30 \mathrm{eV}$, where we have increased the postcollision delay time by at least a factor of 2 , and this had no significant effect on the measured cross section.

The total cross section for the $2^{3} S$ state is presented in Fig. 3. The previous measurements [6,7] and the two contemporary scattering theories, the CCC [2] and RMPS [4]

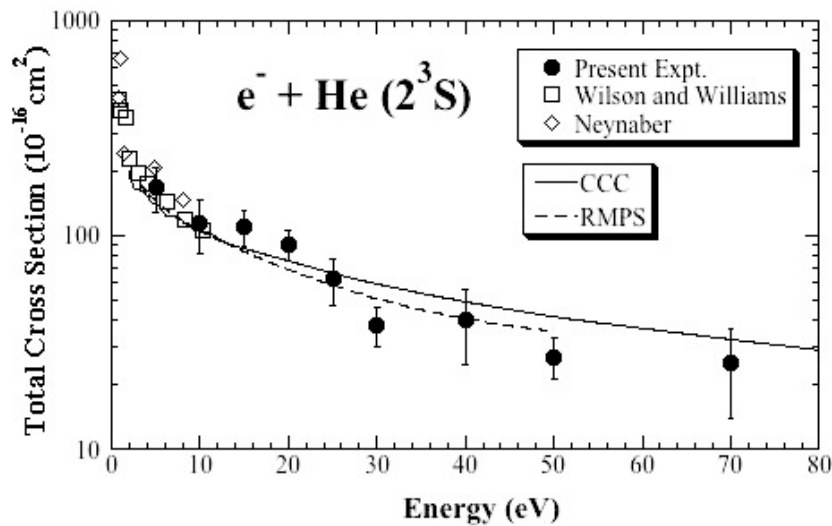

FIG. 3. Total cross section for electrons scattered from metastable $\mathrm{He}\left(2^{3} S\right)$ : $(\bigcirc)$ present results; $(\diamond)$ Neynaber et al. [6]; ( $\left.\square\right)$ Wilson and Williams [7] (normalized to the CCC calculation); (solid line) the convergent close coupling [2] result; (dashed line) the $R$ matrix with pseudostate calculations [4].

calculations, are also shown. Of the previous measurements, only those of [6] are absolute, the data of [7] having been normalized to the CCC calculation. Where the measurements overlap $(\leq 10 \mathrm{eV})$, there is good agreement with the previous data, although the uncertainties in the present measurements are significantly smaller, typically $15 \%-$ $20 \%$. There is also good general agreement with both theoretical calculations across the entire energy range. Given the discrepancies between experiment and theory for this scattering process, this is a significant result, as we shall demonstrate.

The total electron scattering cross section for the metastable helium atoms is dominated at low energies by the contribution from the lowest resonance transition, namely, the electronic excitation from the $2^{3} S$ state to the $2^{3} P$ state. The RMPS calculations [4] indicate that, for a broad range of energies above about $4 \mathrm{eV}$, this excitation cross section exceeds that for elastic scattering. There have been a number of measurements of this excitation cross section, using optical detection techniques, by the Wisconsin group $[17,18]$, with the most recent measurements from this group varying by about a factor of 2 . This was highlighted by Bartschat [4], whose theoretical estimate using the $R$-matrix approach appeared to favor the earlier measurement [18] of the Wisconsin group over the latest one [19].

The present measurements of the total $\mathrm{He}\left(2^{3} S\right)$ cross section can help to shed some light on this issue of the integral cross section for the $2^{3} S-2^{3} P$ transition. In Fig. 4 we show the two measurements from the Wisconsin group and the results of both the RMPS and CCC theories, which are essentially identical to each other and are in better agreement with the earlier, and smaller, cross section result. Given the huge oscillator strength associated with this transition, it is most likely that even a reasonably unsophisticated perturbative calculation would predict the correct cross section. While we do not show it in Fig. 4, 


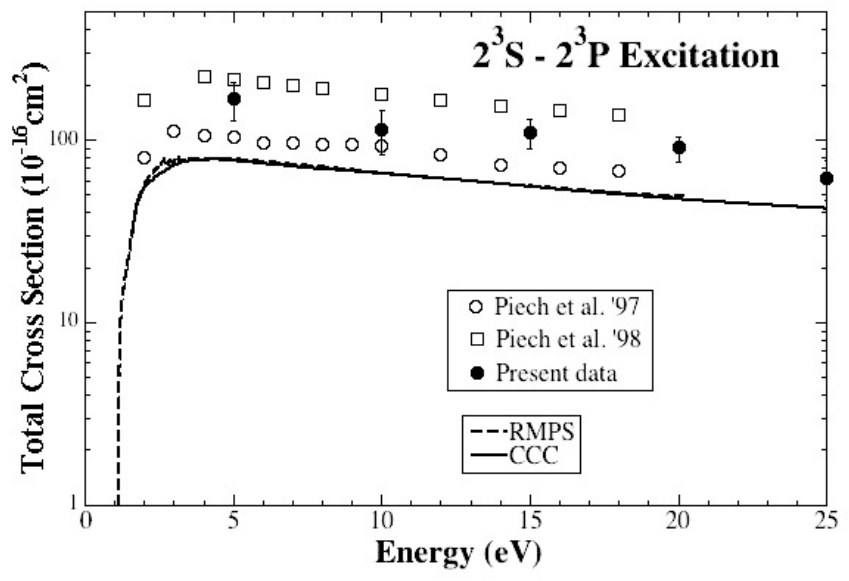

FIG. 4. The measured integral excitation cross section for the $2^{3} S-2^{3} P$ transition (O) [18], ( $\square$ ) [19], compared with (dashed line) the RMPS calculation [4], (solid line) the convergent close coupling result [2], and (O) the present total scattering cross section measurements for the $2^{3} S$ state.

Bartschat [4] demonstrates that a total of four calculations, from an early eikonal approximation to several versions of the $R$-matrix approach, and the $\mathrm{CCC}$ technique all give essentially the same result, which favors the lower excitation cross section. The present total cross section result, also shown in Fig. 4, should, by definition, be larger than any partial cross section that contributes to it. Thus our measurements also strongly favor the earlier Wisconsin result [18], which is smaller than the present total cross section, over the later one [19].

In summary, we have demonstrated how laser-cooling and trapping techniques can be used to greatly enhance the number density and the quality of a metastable helium $\left(2^{3} S\right)$ source for electron collision experiments. This source has been used to measure absolute total scattering cross sections at energies between 5 and $70 \mathrm{eV}$, greatly extending the range of previous measurements and providing a comparison with contemporary scattering theory, where the agreement is seen to be reasonably good. While the agreement with theory at the total scattering level for scattering from the $2^{3} S$ excited state cannot resolve the discrepancies alluded to earlier between experiment and theory for the ionization cross section, the present total cross section for the $2^{3} S$ state clearly supports the magnitude of the theoretical calculations for the dominant $2^{3} S-2^{3} P$ excitation. Further work is planned to attempt to resolve the issue for the ionization cross section and to extend our measurements to scattering from the $2^{3} \mathrm{P}$ excited state.
It is a pleasure to acknowledge discussions with Klaus Bartschat, Igor Bray, and Dima Fursa and to thank them for providing tabulated data. This research is supported by the Australian Research Council through Grant No. DP0343794.

*Permanent address: Physics Department, University of Auckland, Auckland, New Zealand.

${ }^{\dagger}$ Corresponding author.

Electronic address: Stephen.buckman@anu.edu.au

[1] L. G. Christophorou and J. K. Olthoff, Adv. At. Mol. Opt. Phys. 44, 155 (2001).

[2] D. V. Fursa and I. Bray, Phys. Rev. A 52, 1279 (1995); J. Phys. B 30, 757 (1997); 36, 1663 (2003); I. Bray and D. V. Fursa, J. Phys. B 28, L197 (1995); http://atom.murdoch.edu.au/CCC-WWW.

[3] K. Bartschat, J. Phys. B 35, L527 (2002).

[4] K. Bartschat, J. Phys. B 31, L469 (1998); (private communication).

[5] I. Bray, D. V. Fursa, A. S. Kheifets, and A. T. Stelbovics, J. Phys. B 35, R117 (2002).

[6] R. H. Neynaber, S. M. Trujillo, L. L. Marino, and E. W. Rothe, Proceedings of the III International Conference on Photonic, Electronic, and Atomic Collisions (ICPEAC) (New-Holland, Amsterdam, 1964), p. 1089.

[7] W. G. Wilson and W. L. Williams, J. Phys. B 9, 423 (1976).

[8] S. Trajmar and J. Nickel, Adv. At. Mol. Opt. Phys. 30, 45 (1993).

[9] C. C. Lin and L. W. Anderson, Adv. At. Mol. Opt. Phys. 29, 1 (1992).

[10] M. Johnston, K. Fujii, J. Nickel, and S. Trajmar, J. Phys. B 29, 531 (1996).

[11] J. B. Boffard, M. E. Lagus, L. W. Anderson, and C. C. Lin, Rev. Sci. Instrum. 67, 2738 (1996).

[12] R. S. Schappe, P. Feng, L. W. Anderson, C. C. Lin, and T. Walker, Europhys. Lett. 29, 439 (1995).

[13] J.A. MacAskill, W. Kedzierski, J. W. McConkey, J. Domyslawska, and I. Bray, J. Electron Spectrosc. Relat. Phenom. 123, 173 (2002).

[14] R. S. Schappe, T. Walker, L. W. Anderson, and C. C. Lin, Phys. Rev. Lett. 76, 4328 (1996); M. L. Keeler, L. W. Anderson, and C. C. Lin, Phys. Rev. Lett. 85, 3353 (2000).

[15] K. G. H. Baldwin, Contemp. Phys. 46, 105 (2005).

[16] C. J. Dedman, K. G. H. Baldwin, and M. Colla, Rev. Sci. Instrum. 72, 4055 (2001).

[17] A. Browaeys et al., Eur. Phys. J. D 8, 199 (2000).

[18] G. A. Piech, M. E. Lagus, L. W. Anderson, C. C. Lin, and M. R. Flannery, Phys. Rev. A 55, 2842 (1997).

[19] G. A. Piech, J. E. Chilton, L. W. Anderson, and C. C. Lin, J. Phys. B 31, 859 (1998). 